\title{
Establishing the minimum clinically important difference in Neck Disability Index and modified Japanese Orthopaedic Association scores for adult cervical deformity
}

\author{
Alex Soroceanu, MD, MPH, FRCSC, ${ }^{1}$ Justin S. Smith, MD, PhD, ${ }^{2}$ Darryl Lau, MD, ${ }^{3}$ \\ Michael P. Kelly, MD, MSc, ${ }^{4}$ Peter G. Passias, MD, ${ }^{5}$ Themistocles S. Protopsaltis, MD, ${ }^{5}$ \\ Jeffrey L. Gum, MD, ${ }^{6}$ Virginie Lafage, PhD, ${ }^{7}$ Han-Jo Kim, MD, ${ }^{7}$ Justin K. Scheer, MD, ${ }^{3}$ \\ Munish Gupta, MD, ${ }^{4}$ Gregory M. Mundis Jr., MD, ${ }^{8}$ Eric O. Klineberg, MD, ${ }^{9}$ Douglas Burton, MD, ${ }^{10}$ \\ Shay Bess, MD, ${ }^{11}$ Christopher P. Ames, MD, ${ }^{3}$ and the International Spine Study Group \\ ${ }^{1}$ Department of Orthopaedic Surgery, University of Calgary, Alberta, Canada; ${ }^{2}$ Department of Neurosurgery, University of Virginia \\ Health System, Charlottesville, Virginia; ${ }^{3}$ Department of Neurological Surgery, University of California, San Francisco, California; \\ ${ }^{4}$ Department of Orthopaedic Surgery, Washington University, St. Louis, Missouri; ${ }^{5}$ Department of Orthopaedic Surgery, New York \\ University, New York, New York; 'Department of Orthopaedic Surgery, Norton Leatherman Spine Center, Louisville, Kentucky; \\ ${ }^{7}$ Department of Orthopaedic Surgery, Hospital for Special Surgery, New York, New York; ${ }^{8}$ Department of Orthopaedic Surgery, \\ Scripps, San Diego, California; ' ${ }^{D}$ epartment of Orthopaedic Surgery, University of California, Davis, Sacramento, California; \\ ${ }^{10}$ Department of Orthopaedic Surgery, University of Kansas, Kansas City, Kansas; and ${ }^{11}$ Department of Orthopaedic Surgery, \\ Denver International Spine Center, Denver, Colorado
}

OBJECTIVE It is being increasingly recognized that adult cervical deformity (ACD) is correlated with significant pain, myelopathy, and disability, and that patients who undergo deformity correction gain significant benefit. However, there are no defined thresholds of minimum clinically important difference (MCID) in Neck Disability Index (NDI) and modified Japanese Orthopaedic Association (mJOA) scores.

METHODS Patients of interest were consecutive patients with $A C D$ who underwent cervical deformity correction. ACD was defined as C2-7 sagittal Cobb angle $\geq 10^{\circ}$ (kyphosis), C2-7 coronal Cobb angle $\geq 10^{\circ}$ (cervical scoliosis), C2-7 sagittal vertical axis $\geq 4 \mathrm{~cm}$, and/or chin-brow vertical angle $\geq 25^{\circ}$. Data were obtained from a consecutive cohort of patients from a multiinstitutional prospective database maintained across 13 sites. Distribution-based MCID, anchor-based MCID, and minimally detectable measurement difference (MDMD) were calculated.

RESULTS A total of 73 patients met inclusion criteria and had sufficient 1-year follow-up. In the cohort, 42 patients $(57.5 \%)$ were female. The mean age at the time of surgery was 62.23 years, and average body mass index was 29.28. The mean preoperative NDI was 46.49 and mJOA was 13.17. There was significant improvement in NDI at 1 year (46.49 vs 37.04; $p=0.0001$ ). There was no significant difference in preoperative and 1-year mJOA (13.17 vs 13.7; $p=0.12$ ). Using multiple techniques to yield MCID thresholds specific to the ACD population, the authors obtained values of 5.42 to 7.48 for the NDI, and 1.00 to 1.39 for the mJOA. The MDMD was 6.4 for the NDI, and 1.8 for the mJOA. Therefore, based on their results, the authors recommend using an MCID threshold of 1.8 for the MJOA, and 7.0 for the NDI in patients with ACD.

CONCLUSIONS The ACD-specific MCID thresholds for NDI and mJOA are similar to the reported MCID following surgery for degenerative cervical disease. Additional studies are needed to verify these findings. Nonetheless, the findings here will be useful for future studies evaluating the success of surgery for patients with ACD undergoing deformity correction.

https://thejns.org/doi/abs/10.3171/2020.3.SPINE191232

KEYWORDS cervical deformity; Neck Disability Index; modified Japanese Orthopaedic Association; minimum clinically importance difference

\footnotetext{
ABBREVIATIONS $A C D=$ adult cervical deformity; $A S D=$ adult spinal deformity; $E Q-V A S=E Q$ visual analog scale; $M C I D=$ minimum clinically important difference; $M D M D$ $=$ minimally detectable measurement difference; $\mathrm{mJOA}=$ modified Japanese Orthopaedic Association; NDI = Neck Disability Index; PRO = patient-reported outcome; ROC = receiver operating characteristic; $\mathrm{SEM}=$ standard error of measurement.
}

SUBMITTED October 13, 2019. ACCEPTED March 30, 2020.

INCLUDE WHEN CITING Published online May 29, 2020; DOI: 10.3171/2020.3.SPINE191232. 
$\mathrm{T}$ HE pathogenesis and surgical correction of thoracolumbar deformity in patients with adult spinal deformity (ASD) is now a well-studied entity. There are numerous landmark studies and published literature describing its surgical treatment and patient outcomes, resulting in a validated classification system. ${ }^{1}$ On the other hand, adult cervical deformity (ACD) is a more recently characterized pathology, with a newly developed and validated classification system. ${ }^{2}$ Recent studies have demonstrated that ACD, defined as the disruption of normal cervical alignment in the sagittal and/or coronal plane, may be present in up to $53 \%$ of patients with thoracolumbar malalignment. ${ }^{3}$ In fact, the relationship between cervical sagittal alignment and patient-reported outcome (PRO) questionnaires has been increasingly recognized as a significant factor. ${ }^{4,5}$

PRO questionnaires are commonly used to objectively measure a patient's functional status and disability at various follow-up time points; disease-specific PRO questionnaires can be used to assess and measure the effectiveness of certain medical and/or surgical interventions. The minimum clinically important difference (MCID) represents the smallest change in PRO that corresponds to meaningful improvement. MCID is dependent on specific pathology and the appropriateness of PRO questionnaires. Patients with ACD have many inherently distinct features and characteristics compared to the degenerative cervical conditions used in the development of the cervical-specific PRO questionnaires. Whereas MCID thresholds have been established for adult thoracolumbar deformity and for degenerative cervical spine pathologies, to our knowledge there are no established MCID thresholds specific to the ACD population.

Therefore, the purpose of this study was to establish MCID thresholds for the Neck Disability Index (NDI) and modified Japanese Orthopaedic Association (mJOA) in patients with ACD undergoing surgical intervention for deformity correction.

\section{Methods \\ Study Data}

All patients included in this study had the diagnosis of ACD and underwent surgery primarily for deformity correction. Study data were obtained from a multiinstitutional prospective database of consecutively enrolled patients with ACD undergoing surgical correction of their deformity. Patients were enrolled at 13 institutions across the United States. The study was approved by the institutional review boards of all participating centers.

\section{Patients and Follow-Up}

The general inclusion criteria for the database were as follows: age $>18$ years and presence of cervical deformity, as defined by at least 1 of the following: C2-7 sagittal Cobb angle $\geq 10^{\circ}$ (cervical kyphosis), C2-7 coronal Cobb angle $\geq 10^{\circ}$ (cervical scoliosis), C2-7 sagittal vertical axis $\geq 4 \mathrm{~cm}$, and/or chin-brow vertical angle $\geq 25^{\circ}$. Exclusion criteria included the presence of active infection or malignancy. Patients underwent ACD correction surgery at their respective centers. All patients were evaluated preopera- tively, and 3 months, 6 months, and 1 year after surgery by using standardized forms. For this study, changes in PRO were calculated based on the difference between preoperative and 1-year postoperative scores. In addition to the above inclusion criteria, the present study only included patients who completed baseline and 1-year follow-up PROs. For calculations pertaining to the mJOA, patients without myelopathy (baseline mJOA = 18) were excluded.

\section{Distribution-Based MCID}

All statistical analysis was performed using Stata. The principle of distribution-based methods is to compare a change in PRO scores to a measure of variability. The use of one standard error of measurement (1 SEM) and 0.5 SD as an approximation of the MCID have been proposed in the literature. ${ }^{6-8}$ In this study, both methods were used to determine the MCID for the NDI and mJOA. For the 1 SEM method, the SEM was calculated using the following equation: $\mathrm{SEM}=\mathrm{SD} \times(1-\mathrm{R})^{1 / 2}$, where $\mathrm{SD}$ represents the standard deviation at baseline and $\mathrm{R}$ represents the reliability coefficient.

\section{Anchor-Based MCID}

The goal of anchor-based methods is to compare a change in PRO scores to another measure of change, an external criterion called an anchor. Patients' response to a global assessment scale is often used as an anchor to determine MCID. ${ }^{6-8}$ In this study the improvement on the EQ visual analog scale (EQ-VAS) was used as an anchor and divided into 4 groups based on quartiles $(<25$ th percentile, "worsened"; 25th-50th percentile, "unchanged"; 51st-75th percentile, "slight improvement"; and > 75th percentile, "marked improvement"). Anchor-based MCID thresholds were computed using receiver operating characteristic (ROC) curve analysis.

\section{Minimally Detectable Measurement Difference}

More recently, the concept of minimally detectable measurement difference (MDMD) has been described. The MDMD determines the smallest change in an outcome that can be measured reliably; in other words, the smallest change that is larger than measurement error. The MDMD was calculated for both the mJOA and NDI using the methodology reported by Spratt. ${ }^{9}$ In the event that the distribution and anchor-based thresholds were both lower than the MDMD, the MDMD score was used to approximate the MCID.

\section{Results}

A total of 122 patients were eligible for 1-year followup; 73 patients $(60 \%)$ had complete preoperative and 1 -year postoperative health-related quality of life data and were included in the study. The mean age at the time of surgery was 62.27 years, and the average body mass index was 29.28 (Table 1). Among the cohort, 42 patients $(57.5 \%)$ were female and the mean Charlson Comorbidity Index was 0.63 . Almost half of cases had undergone prior surgery $(45 \%)$. Blood loss and operating time were 828.5 $\mathrm{ml}$ and 357.98 minutes, respectively. 
TABLE 1. Demographic, comorbidity, and surgical information in 73 patients with ACD

\begin{tabular}{lcc}
\hline \multicolumn{1}{c}{ Characteristic } & Mean or $\%$ & SD \\
\hline Age $(\mathrm{yrs})$ & 62.27 & 10.66 \\
\hline $\mathrm{BMI}$ & 29.28 & 7.84 \\
\hline $\mathrm{CCl}$ & 0.63 & 1.03 \\
\hline Female & $57.5 \%$ & \\
\hline Prior cervical spine surgery & $45 \%$ & \\
\hline Blood loss (ml) & 828.50 & 853.86 \\
\hline Surgical time (mins) & 357.98 & 225.63 \\
\hline
\end{tabular}

$\mathrm{BMI}=$ body mass index $; \mathrm{CCl}=$ Charlson Comorbidity Index .

\section{Preoperative and Postoperative PROs}

As shown in Table 2, the mean preoperative NDI was 46.49 and mJOA was 13.17. There was significant improvement in NDI at 1 year $(46.49$ vs 37.04$)(p=0.0001)$. There was no significant difference in preoperative and 1 -year mJOA (13.17 vs 13.7) $(\mathrm{p}=0.12)$.

\section{Distribution-Based MCID Thresholds}

Using the 0.5 SD method, the MCID for the NDI was estimated to be 7.48, and the MCID for the MJOA was estimated to be 1.39. The SEM-computed MCID gives us an estimate of 5.42 for the NDI, and of 1.24 for the mJOA. Results of the MDMD for each PRO can also be found in Table 3. According to our results, the MDMD was 6.41 for the NDI, and 1.82 for the mJOA.

When looking at the NDI response to the anchor, patients who were classified as "worsened" based on the EQVAS improved on average by $1.75( \pm 12.09)$ points on the NDI. On the other hand, those classified as "unchanged" improved by $6.87( \pm 15.46)$ points, patients classified as "slightly improved" saw an improvement of $10.63( \pm$ 10.73 ) points, and those classified as "markedly improved" showed a difference of 20.72 ( \pm 14.09$)$ points. When looking at the $\mathrm{mJOA}$ response to the anchor, patients classified as "worsened" saw a decrease of $1.42( \pm 2.11)$ points, those classified as "unchanged" had an improvement of 0.55 ( \pm 2.76$)$ points, those classified as "slightly improved" showed a difference of $0.88( \pm 2.13)$ points, and those classified as "markedly improved" had a difference of 1.21 ( \pm 2.99) points. Results are summarized in Table 4.

Discrete MCID thresholds computed using ROC curve analysis are presented in Table 5. This yielded an MCID of 7.00 for the NDI, and 1.00 for the mJOA.

\section{Discussion}

Using multiple techniques to yield MCID thresholds

\section{TABLE 2. Preoperative and 1-year follow-up NDI and mJOA} scores

\begin{tabular}{lccl}
\hline PRO & Mean Preop Score & Mean 1-Yr Postop Score & $p$ Value \\
\hline NDI & 46.49 & 37.04 & 0.0001 \\
\hline mJOA & 13.17 & 13.7 & 0.12 \\
\hline
\end{tabular}

TABLE 3. Distribution-based MCID thresholds

\begin{tabular}{cccc}
\hline & & \multicolumn{2}{c}{ Distribution-Based } \\
\cline { 3 - 4 } PRO & MDMD & SEM Method & 0.5 SD Method \\
\hline NDI & 6.41 & 5.42 & 7.48 \\
\hline mJOA & 1.82 & 1.24 & 1.39 \\
\hline
\end{tabular}

specific to the ACD population, we obtained values of 5.42 to 7.48 for the NDI, and 1.24 to 1.39 for the mJOA. The MDMD was 6.4 for the NDI, and 1.8 for the mJOA. Therefore, based on our results, we recommend using an MCID threshold of 1.8 for the mJOA, and 7.0 for the NDI in patients with ACD. Using these thresholds, $60 \%$ of our cohort met the criteria of reaching MCID for the NDI and $29 \%$ met the criteria of reaching MCID for the mJOA 1 year postsurgery.

Although this study is the first to determine MCID thresholds specific to the ACD population, several studies have previously established these thresholds for degenerative spinal pathologies. Using different methods, Auffinger et al. found MCID values of 4.83-13.39 for NDI in a cohort of patients undergoing anterior cervical discectomy and fusion for cervical spondylotic myelopathy. ${ }^{10}$ The same authors later proposed an NDI MCID of 2.72-12.08 in patients with degenerative cervical pathologies. ${ }^{11} \mathrm{An}$ NDI MCID of 7.5 was suggested by Carreon et al. for patients undergoing cervical fusion surgery for degenerative pathologies. ${ }^{12}$ Similarly, Young et al. recommended an MCID of 7.5 in patients with mechanical neck pain undergoing physiotherapy. ${ }^{13}$ The NDI MCIDs established in patients with ACD are surprisingly similar to some of the thresholds suggested by previous authors for degenerative cervical spine pathologies. This is important because it may suggest that patients with ACD with high preoperative NDI secondary to severe deformity can gain significant clinical benefit even with small changes in NDI score.

In regard to $\mathrm{mJOA}$ MCID, the values yielded by distribution- and anchor-based analysis in our patient population were both lower than the MDMD. The MDMD determines the smallest change in an outcome that can be reliably measured, meaning the smallest change that is larger than measurement error. By comparison, the MCID relies

TABLE 4. NDI and mJOA response to anchor

\begin{tabular}{|c|c|}
\hline Anchor Score & Mean (SD) \\
\hline \multicolumn{2}{|c|}{ NDI response to anchor } \\
\hline 1 & $-1.75(12.09)$ \\
\hline 2 & $-6.87(15.46)$ \\
\hline 3 & $-10.63(10.73)$ \\
\hline 4 & $-20.72(14.09)$ \\
\hline \multicolumn{2}{|c|}{ mJOA response to anchor } \\
\hline 1 & $-1.42(2.11)$ \\
\hline 2 & $0.55(2.76)$ \\
\hline 3 & $0.88(2.13)$ \\
\hline 4 & $1.21(2.99)$ \\
\hline
\end{tabular}


TABLE 5. Discrete MCID thresholds computed using ROC curve analysis

\begin{tabular}{ccc}
\hline PRO & ROC MCID & AUC \\
\hline NDI & 7.000 & $0.719(0.601-0.837)$ \\
\hline mJOA & 1.000 & $0.619(0.479-0.758)$ \\
\hline
\end{tabular}

$\mathrm{AUC}=$ area under the curve.

on patients being classified as "improved" versus "not improved," and does not take into account the measurement error of the outcome measure itself. Because the MDMD concept has been introduced and is more widely accepted, efforts should be made to ensure that MCID thresholds are larger than the measurement error calculated by the MDMD. As such, we propose to use the MDMD (1.8) as an MCID threshold for the mJOA in patients with ACD.

Although this is the first study to look at the mJOA MCID threshold specific to the ACD population, several other studies have previously aimed to establish an mJOA MCID in the context of degenerative cervical pathology. Our result of an MCID of 1.8 specific to patients with ACD is similar to MCID thresholds proposed for degenerative conditions. Zhou et al. proposed an mJOA MCID of 1.25-3.07 in a cohort of patients with cervical spondylotic myelopathy. ${ }^{14}$ Similarly, Tetreault et al. looked at establishing MCID thresholds for the mJOA in patients with degenerative myelopathy by using distributionbased techniques, anchor-based techniques, and a survey of spine surgeons. Their recommended MCID was 1-2. These authors also suggested that, in the degenerative myelopathy population, different MCID thresholds should be used for patients who have mild, moderate, and severe myelopathy at baseline. According to their results, patients with mild myelopathy had an MCID of 1 , and those with severe myelopathy had an MCID of $3 .{ }^{15}$ Our sample size did not allow us to verify if this theory holds in the ACD population. Future research should investigate whether having different mJOA MCID thresholds according to baseline myelopathy severity (as suggested for the degenerative cervical myelopathy population) also translates to the ACD population.

This study has a number of limitations, including the anchor used for the anchor-based MCID calculation. Even though the EQ-VAS has a statistically significant correlation with both the NDI and the mJOA, it is not a categorical measure, and therefore had to be transformed into arbitrary categories based on quartiles. Another potential limitation is the small number of patients in this study, which limits the power of the analysis. However, it is only more recently that ACD is being recognized as a main driver of pain and disability, and therefore there is a paucity of studies examining outcomes in this patient population. In addition, this study is among the largest series of patients with ACD in the published literature. Despite these limitations, our study is the first to establish MCID thresholds specific to the ACD population. These newly identified thresholds specific for functional improvement based on PRO should be considered and used in future studies evaluating the success of surgery for patients with ACD.

\section{Conclusions}

Based on the findings in this study, we recommend using an MCID threshold of 1.8 for the mJOA, and 7.0 for the NDI for patients with ACD. The NDI and mJOA MCID established in patients with ACD are similar to reported thresholds suggested by previous authors for degenerative cervical spine pathologies. These new ACD-specific thresholds will be useful in future studies evaluating the success and utility of surgery for patients with ACD undergoing deformity correction.

\section{References}

1. Terran J, Schwab F, Shaffrey CI, et al. The SRS-Schwab adult spinal deformity classification: assessment and clinical correlations based on a prospective operative and nonoperative cohort. Neurosurgery. 2013;73(4):559-568.

2. Ames CP, Smith JS, Eastlack R, et al. Reliability assessment of a novel cervical spine deformity classification system. $J$ Neurosurg Spine. 2015;23(6):673-683.

3. Jalai CM, Passias PG, Lafage V, et al. A comparative analysis of the prevalence and characteristics of cervical malalignment in adults presenting with thoracolumbar spine deformity based on variations in treatment approach over 2 years. Eur Spine J. 2016;25(8):2423-2432.

4. Gum JL, Glassman SD, Douglas LR, Carreon LY. Correlation between cervical spine sagittal alignment and clinical outcome after anterior cervical discectomy and fusion. Am J Orthop. 2012;41(6):E81-E84.

5. Scheer JK, Passias PG, Sorocean AM, et al. Association between preoperative cervical sagittal deformity and inferior outcomes at 2-year follow-up in patients with adult thoracolumbar deformity: analysis of 182 patients. J Neurosurg Spine. 2016;24(1):108-115.

6. Copay AG, Subach BR, Glassman SD, et al. Understanding the minimum clinically important difference: a review of concepts and methods. Spine J. 2007;7(5):541-546.

7. Doganay Erdogan B, Leung YY, Pohl C, et al. Minimal clinically important difference as applied in rheumatology: an OMERACT Rasch Working Group systematic review and critique. J Rheumatol. 2016;43(1):194-202.

8. Wells G, Beaton D, Shea B, et al. Minimal clinically important differences: review of methods. J Rheumatol. 2001;28(2):406-412.

9. Spratt KF. Patient-level minimal clinically important difference based on clinical judgment and minimally detectable measurement difference: a rationale for the SF-36 Physical Function Scale in the SPORT intervertebral disc herniation cohort. Spine (Phila Pa 1976). 2009;34(16):1722-1731.

10. Auffinger BM, Lall RR, Dahdaleh NS, et al. Measuring surgical outcomes in cervical spondylotic myelopathy patients undergoing anterior cervical discectomy and fusion: assessment of minimum clinically important difference. PLoS One. 2013;8(6):e67408.

11. Auffinger B, Lam S, Shen J, et al. Usefulness of minimum clinically important difference for assessing patients with subaxial degenerative cervical spine disease: statistical versus substantial clinical benefit. Acta Neurochir (Wien). 2013;155(12):2345-2355.

12. Carreon LY, Glassman SD, Campbell MJ, Anderson PA. Neck Disability Index, Short Form-36 Physical Component Summary, and pain scales for neck and arm pain: the minimum clinically important difference and substantial clinical benefit after cervical spine fusion. Spine J. 2010;10(6):469-474.

13. Young BA, Walker MJ, Strunce JB, et al. Responsiveness of the Neck Disability Index in patients with mechanical neck disorders. Spine J. 2009;9(10):802-808. 
14. Zhou F, Zhang Y, Sun Y, et al. Assessment of the minimum clinically important difference in neurological function and quality of life after surgery in cervical spondylotic myelopathy patients: a prospective cohort study. Eur Spine $J$. 2015;24(12):2918-2923.

15. Tetreault L, Nouri A, Kopjar B, et al. The minimum clinically important difference of the modified Japanese Orthopaedic Association scale in patients with degenerative cervical myelopathy. Spine (Phila Pa 1976). 2015;40(21):1653-1659.

\section{Disclosures}

Dr. Ames is an employee of UCSF. He is a consultant for DePuy Synthes, Medtronic, Stryker, Medicrea, K2M, and Biomet Zimmer. He receives royalties from Stryker, Biomet Zimmer Spine, DePuy Synthes, NuVasive, Next Orthosurgical, K2M, and Medicrea. He received educational research grants from Titan Spine, DePuy Synthes, and the International Spine Study Group (ISSG). He serves on the editorial board of Operative Neurosurgery. He has received grant funding from the Scoliosis Research Society (SRS), and he is on the executive committee of the ISSG and Global Spinal Analytics. Dr. Bess is a consultant for K2M Stryker and a patent holder with K2M. He received clinical or research support for the study described (includes equipment or material) from the ISSG Foundation, and he also received support of non-study-related clinical or research efforts that he oversaw from the ISSG Foundation. Dr. Burton received clinical or research support for the study described (includes equipment or material) from DePuy and Pfizer. He is a patent holder with DePuy. He has direct stock ownership in Progenerative Medical, and is a consultant for Bioventus. Dr. Gum is an employee of Norton Healthcare. He is a consultant for Medtronic, DePuy, Stryker, Acuity, K2M, PacMed, and NuVasive. He received clinical or research support for the study described (includes equipment or material) from Intellirod, Integra, Pfizer, and the International Spine Study Group. He has direct stock ownership in Cingulate Therapeutics, and he is a patent holder with Medtronic. He receives royalties from Acuity. Dr. Gupta has direct stock ownership in J\&J (Johnson \& Johnson) and P\&G (Procter \& Gamble). He is a consultant for Medtronic and DePuy. He receives royalties from Innomed and from DePuy, and he also has acted in an advisory capacity for DePuy. He has received support for travel from Medicrea, Alphatec, SRS, DePuy, AO Spine, and Mizuho. OMeGA and AO Spine paid grants to his institution for fellowships, and AO Spine also paid him an honorarium. Dr. Kelly received support of a non-study-related clinical or research effort that he oversaw from DePuy Synthes. Dr. Klineberg is a consultant for DePuy Synthes, Stryker, and Medicrea. He received a fellowship grant and honoraria from AO Spine. Dr. Lafage is a consultant for Globus Medical and receives royalties from NuVasive. She received honoraria from The Permanente Medical Group and DePuy Synthes. She is on the executive committee of the International Spine Study Group. Dr. Mundis is a consultant for NuVasive, K2M, Viseon, and Seaspine. He has direct stock ownership in NuVasive and Viseon. $\mathrm{He}$ receives royalties from NuVasive and K2M. Dr. Passias is a consultant for SpineWave and Medicrea. He is a paid presenter or speaker for Zimmer and Globus Medical. He received clinical or research support for the study described (includes equipment or material) from the Cervical Scoliosis Research Society. He also received support of non-study-related clinical or research efforts that he oversaw from Allosource. Dr. Protopsaltis is a consultant for Globus, Stryker, K2M, NuVasive, Medicrea, and Innovasis. He receives royalties from Altus. Dr. Smith is a consultant for K2M/ Stryker, AlloSource, Cerapedics, Zimmer Biomet, NuVasive, and DePuy Synthes. He received clinical or research support for the study described (includes equipment or material) from DePuy Synthes/ISSG. He also received support of non-study-related clinical or research efforts that he oversaw from DePuy Synthes/ ISSG and AO Spine. Other conflicts include the following: royalties from Zimmer Biomet and NuVasive; fellowship funding from NREF and AO Spine; research support from AO Spine; and stock ownership in Alphatec.

\section{Author Contributions}

Conception and design: Soroceanu, Smith, Ames. Acquisition of data: Soroceanu, Smith, Kelly, Passias, Protopsaltis, Gum, Lafage, Kim, Gupta, Mundis, Klineberg, Burton, Bess, Ames. Analysis and interpretation of data: Soroceanu, Smith, Kelly, Passias, Protopsaltis, Gum, Lafage, Kim, Scheer, Gupta, Klineberg, Ames. Drafting the article: Soroceanu. Critically revising the article: Lau, Soroceanu, Smith, Ames. Reviewed submitted version of manuscript: all authors. Approved the final version of the manuscript on behalf of all authors: Lau. Statistical analysis: Lafage, Scheer. Study supervision: Soroceanu, Bess, Ames.

\section{Correspondence}

Darryl Lau: University of California, San Francisco, CA. darryl. lau@ucsf.edu. 